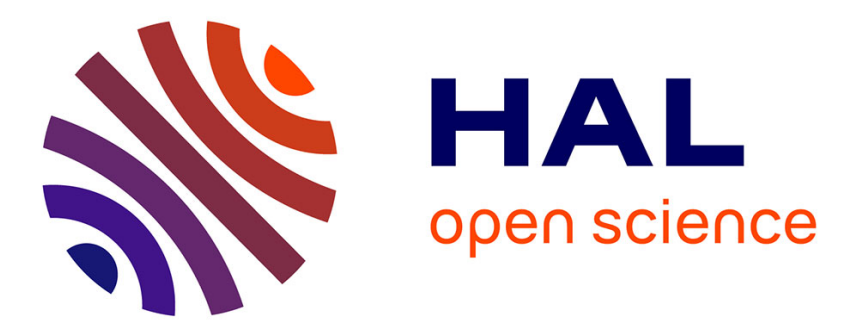

\title{
Phase diagram of thiourea at atmospheric pressure under electric field : a theoretical analysis \\ P. Lederer, C.M. Chaves
}

\section{To cite this version:}

P. Lederer, C.M. Chaves. Phase diagram of thiourea at atmospheric pressure under electric field: a theoretical analysis. Journal de Physique Lettres, 1981, 42 (6), pp.127-130. 10.1051/jphyslet:01981004206012700 . jpa-00231890

\section{HAL Id: jpa-00231890 https://hal.science/jpa-00231890}

Submitted on 1 Jan 1981

HAL is a multi-disciplinary open access archive for the deposit and dissemination of scientific research documents, whether they are published or not. The documents may come from teaching and research institutions in France or abroad, or from public or private research centers.
L'archive ouverte pluridisciplinaire HAL, est destinée au dépôt et à la diffusion de documents scientifiques de niveau recherche, publiés ou non, émanant des établissements d'enseignement et de recherche français ou étrangers, des laboratoires publics ou privés. 


\title{
Phase diagram of thiourea at atmospheric pressure under electric field : a theoretical analysis
}

\author{
P. Lederer $\left(^{*}\right)$ and C. M. Chaves \\ Departamento de Fisica, P.U.C., rua Marquès de São Vicente 225, 20000 Rio de Janeiro, Brazil
}

(Reçu le 15 décembre 1980, révisé le 29 janvier 1981, accepté le 30 janvier 1981)

\begin{abstract}
Résumé. - Nous discutons le diagramme de phase de la thiourée dans le plan $(E, T)$ à la pression atmosphérique $P_{0}$, dans une approximation à une seule harmonique pour la phase modulée. Nous utilisons une approche à la Landau-Ginzburg, du genre de celle qui permet de discuter un point de Lifshitz : la variation thermique du carré du vecteur d'onde de la modulation est gouvernée par la variation thermique de la raideur d'échange négative. Cette théorie permet de comprendre qualitativement et semi-quantitativement le diagramme de phase. Ce dernier possède un point tricritique dans le voisinage d'un point critique isolé.
\end{abstract}

\begin{abstract}
The phase diagram of thiourea in the $(E, T)$ plane at atmospheric pressure $P_{0}$ is discussed in terms of a single harmonic approximation for the modulated phase. We use a Landau-Ginzburg approach of the type used to discuss Lifshitz points ; in this approach the temperature dependence of the square of the modulation wave vector is accounted for by the temperature dependence of the negative exchange stiffness constant. This theory allows qualitative as well as semi-quantitative understanding of the phase diagram, which is shown to exhibit a tricritical point in the close neighbourhood of an isolated critical point.
\end{abstract}

The phase diagram shown in reference [1], figure 3 exhibits the following features :

i) A first-order transition line $T_{\mathrm{H}}(E)$ starts at $190 \mathrm{~K}, E=0$ and terminates at a critical point $T_{\mathrm{M}}=210 \pm 0.4 \mathrm{~K}$ and $E_{\mathrm{M}}=2.285 \pm 10 \mathrm{~V} / \mathrm{mm}$. At this point the derivative $\mathrm{d} T_{\mathrm{H}}(E) / \mathrm{d} E$ appears to be infinite.

ii) A small segment of curve between $T_{M}$ and approximately $212.5 \mathrm{~K}, E=2050 \mathrm{~V} / \mathrm{mm}$ which, upon careful examination of the experimental curves in figure 2 seems to be a segment of weakly first-order transition. If such is the case, as further experiments might confirm, the point $T_{\mathrm{tr}}=212.5 \mathrm{~K}$ is a tricritical point.

iii) For $T>212.5 \mathrm{~K}$, the transition line $T(E)$ is clearly a second-order line which terminates at $T=216 \mathrm{~K}, E=0$.

iv) A commensurate phase, which had been previously studied by neutron diffraction techniques in zero field [2]. It is also striking that the curve $T_{\mathrm{H}}(E)$

$\left({ }^{*}\right)$ On leave of absence from Physique des Solides, Bâtiment 510, Université Paris-Sud, Centre d’Orsay, 91405 Orsay, France. has practically zero curvature up to about $2000 \mathrm{~V} / \mathrm{mm}$. Finally, as already known [3], the modulation wave vector varies with temperature in the modulated phase at zero field, from $q \sim 1 / 7$ at $216 \mathrm{~K}$ to $q=1 / 9$ at $191 \mathrm{~K}$.

The experimental results can be understood in a semi-quantitative way if one takes into account in a consistent way the temperature variation of the modulation wave vector, as is done in the Landau theory of a Lifshitz point [4].

The relevant free energy has the following form $[4,5]$

$$
\begin{aligned}
F=\int\left(\frac{1}{2} A_{0} P_{z}^{2}+\right. & \frac{1}{4} B P_{z}^{4}+\frac{1}{2} \alpha\left(\frac{\partial P_{z}}{\partial x}\right)^{2}+ \\
& \left.+\frac{1}{4} \beta\left(\frac{\partial^{2} P_{z}}{\partial x^{2}}\right)^{2}-E_{z} P_{z}\right) \mathrm{d}^{3} r
\end{aligned}
$$

where $P_{z}$ is the uniaxial polarization and $E$ is the electric field. Here $A_{0}=A_{0}(T, P) ; B$ and $\beta$ are positive constants and $\alpha(T, P)$ is negative. The $\alpha_{i}$ parameters associated with other directions are assumed to be positive and bring no new physics into the problem. 
In zero field, if $\alpha>0$, the line $A_{0}(T, P)=0$ defines the second-order para-ferroelectric transition line $T_{0}(P)$. When $\alpha<0$, the state of equilibrium is spatially non-uniform along the $x$-direction. The Lifshitz point is defined by $A_{0}\left(T_{\mathrm{L}}, P_{\mathrm{L}}\right)=\alpha\left(T_{\mathrm{L}}, P_{\mathrm{L}}\right)=0$. Measurements [1] are done at atmospheric pressure $P_{0} \neq P_{\mathrm{L}}$.

In the simple theory described here, we shall consider only one harmonic for the modulation in the whole sinusoidal phase, so that we expect to describe poorly the region below about $203 \mathrm{~K}$. In particular we shall discuss only briefly the locked phase [2] at $q=1 / 9$ or other fine details of the $q(T)$ curve, which are outside the scope of the simple harmonic approximation. Our purpose is to account for overall gross features of the phase diagram. Within the molecular field approximation, we have

$$
q^{2}(T)=-\alpha(T, P) / \beta .
$$

Experimentally $[3,6] q^{2}(T)$ at zero field is found to be linear in temperature down to about $203 \mathrm{~K}$. In reference [6] it is shown that near $q_{0}=1 / 9,\left(q-q_{0}\right)^{2}$ varies linearly with $T$. The reason for this behaviour, and for the pinning at $q=1 / 9$ is probably linked with the many Fourier components of the modulation, and with Umklapp terms [7] which we neglect in equation (1).

It was shown [4] that the second-order parasinusoidal transition line in zero field is given by

$$
T_{\lambda}(P)=T_{0}(P)+\frac{C \alpha^{2}\left(T_{\lambda}, P\right)}{2 \beta} ;
$$

$T_{0}(P)$ is the extrapolation for $\alpha<0$ of the line $T_{0}(P)$ for $\alpha>0$. $C$ is the Curie-Weiss constant for the susceptibility in the para-electric phase. The ferrosinusoidal transition (in zero field) was shown to be first-order [4], given by

$$
T_{\mathrm{H}}(P)=T_{0}(P)-2.2 C \frac{\alpha^{2}\left(T_{\mathrm{H}}, P\right)}{\beta} .
$$

The projection of the line of tricritical points on the $T, P$, diagram [5] is

$$
T_{\mathrm{tr}}(P)=T_{0}(P)+\frac{3}{8} C \frac{\alpha^{2}\left(T_{\mathrm{tr}} P\right)}{\beta} .
$$

We can rewrite (2), (3), (4) as

$$
T_{i}=T_{0}(P)+\mu_{i} C \frac{\alpha^{2}\left(T_{i}, P\right)}{\beta}
$$

where $i$ can be $\lambda, \operatorname{tr}$, or $\mathrm{H}$ and $\mu_{i}$ is defined in an obvious way.

As a result, the following relations hold :

$$
\frac{T_{i}-T_{0}(P)}{T_{j}-T_{0}(P)}=\frac{\mu_{i}}{\mu_{j}}\left(\frac{q\left(T_{i}\right)}{q\left(T_{j}\right)}\right)^{4}
$$

Using equation (6) and the experimental values for $T_{\lambda}$ and $T_{\mathrm{H}}$ in zero field, as well as the experimental values for $q\left(T_{\lambda}\right)$ and $q\left(T_{\mathrm{H}}\right)$ one determines $T_{0}(P)$, and one obtains a theoretical estimate for $T_{\mathrm{tr}}$. Alternatively, using the experimental values for $T_{\mathrm{tr}}$ one has a second independent determination for $T_{0}$. One thus obtains

and

$$
T_{\mathrm{tr}}^{\text {theor }}=213 \mathrm{~K}
$$

or

$$
\begin{aligned}
& T_{0}=206 \mathrm{~K} \\
& T_{0}=208.5 \mathrm{~K} .
\end{aligned}
$$

Given the naive approach used, we may consider the agreement between theory and experiment as remarkable. Furthermore, one computes easily the derivative $\mathrm{d} T_{\lambda}(E) / \mathrm{d} E$ at $E=E_{\mathrm{tr}}$. One finds

$$
\begin{aligned}
\left.\frac{\mathrm{d} T_{\lambda}}{\mathrm{d} E}\right|_{T_{\mathrm{tr}}} & =-\frac{2 C \sqrt{6 B \beta}}{\alpha\left(T_{\mathrm{tr}}\right)}\left(1-2 C \frac{\alpha\left(T_{\mathrm{tr}}\right)}{\beta} y\right)^{-1} \\
& =-\frac{10}{9} \frac{T_{\mathrm{tr}}-T_{0}}{E_{\mathrm{tr}}}\left(1-2 C \frac{\alpha\left(T_{\mathrm{tr}}\right)}{\beta} y\right)^{-1}
\end{aligned}
$$

where $y=\mathrm{d} \alpha /\left.\mathrm{d} T\right|_{T_{\mathrm{tr}}}$.

Equation (8) shows that one cannot have

$$
\left.\frac{\mathrm{d} T_{\lambda}}{\mathrm{d} E}\right|_{E_{\mathrm{tr}}}
$$

infinite unless the temperature dependence of $\alpha$ is taken into account. The factor

$$
S=\left(1-2 C \frac{\alpha\left(T_{\mathrm{tr}}\right)}{\beta} y\right)^{-1}
$$

may be considered as an enhancement factor of the slope $\mathrm{d} T_{\lambda} / \mathrm{d} E$ at $T_{\mathrm{tr}}$ as compared with its value very near the Lifshitz point, where the enhancement factor is one $(\alpha \rightarrow 0)$. Taking the experimental value

$$
\left.\frac{\mathrm{d} T_{\lambda}}{\mathrm{d} E}\right|_{E_{\mathrm{tr}}}=(10 \pm 5) \cdot 10^{-3} \mathrm{~K} \cdot \mathrm{V}^{-1} \cdot \mathrm{mm}
$$

the enhancement factor is found to be

$$
S=3.3 \pm 1.5 \text {. }
$$

The enhancement factor may be evaluated independently from the curvature of the $T_{\mathrm{H}}(E)$ curve near $E=0$.

The first derivative is

$$
\begin{aligned}
\left.\frac{\mathrm{d} T_{\mathrm{H}}}{\mathrm{d} E}\right|_{T_{\mathrm{H}}^{\mathrm{o}}} & =\frac{5 C \sqrt{2.2 B \beta}}{\alpha\left(T_{\mathrm{H}}\right)\left(1+\frac{9}{2} \frac{\alpha\left(T_{\mathrm{H}}\right)}{\beta} C y\right)} \\
\left.\frac{\mathrm{d} T_{\mathrm{H}}}{\mathrm{d} E}\right|_{T_{\mathrm{H}}^{\mathrm{O}}} & =\frac{1.7 a^{2}}{(a+1.7)} \frac{T_{\mathrm{tr}}-T_{0}}{E_{\mathrm{tr}}} \\
& =(4 \pm 0.2) \cdot 10^{-3}
\end{aligned}
$$


where

$a=\alpha\left(T_{\mathrm{tr}}\right) / \alpha\left(T_{\mathrm{H}}^{0}\right) \equiv\left(\frac{q\left(T_{\mathrm{tr}}\right)}{q\left(T_{\mathrm{H}}^{0}\right)}\right)^{2} \quad$ and $\quad T_{\mathrm{H}}^{0}=T_{\mathrm{H}}(E=0)$.

Equation $\left(9^{\prime}\right)$ compares reasonably well with the experimental data $7 \times 10^{-3}$, without adjustable parameter. It is tempting to ascribe the discrepancy to the many Fourier component effects.

We also find

$$
\begin{aligned}
& \left.\frac{\mathrm{d}^{2} T_{\mathrm{H}}}{\mathrm{d} E^{2}}\right|_{E=0}= \\
& =-\left.4 \frac{\left(x^{2}-x / 3-1 / 5\right)}{1+9 x / 4}\left(\frac{1}{T_{\mathrm{H}}^{0}-T_{0}}\right)\left(\frac{\mathrm{d} T_{\mathrm{H}}}{\mathrm{d} E}\right)^{2}\right|_{T_{\mathbf{H}}^{0}}
\end{aligned}
$$

where $x=(S-1) / a S$.

As noticed above, $\mathrm{d}^{2} T_{\mathrm{H}} / \mathrm{d} E^{2}$ is very nearly zero at $E=0$, so that this determines $x$, whence we find $S=4.2$, in satisfactory agreement with the estimate above. As for the critical point at $T_{\mathrm{M}}$, its existence is linked to the maximum in the $T_{\mathrm{H}}(E)$ curve. Although the $T_{\mathrm{H}}(E)$ curve is in principle a first-order transition line, it is straightforward to check, for example using Clapeyron equation, that a maximum in the $T_{\mathrm{H}}(E)$ curve is a point with zero entropy jump [8]. Thus $T_{M}$ is an isolated critical point at the maximum of the $T_{\mathrm{H}}(E)$ curve. In the $E, T, P$ space, the surface of first-order transitions is cut in two by a line of isolated critical points which crosses the plane $E, T, P_{0}$ at $T_{\mathrm{M}}$. The interesting aspect of the physics of thiourea is that $T_{\mathrm{M}}$ and $T_{\mathrm{tr}}$ are not far from one another. Nothing prevents them from collapsing into a single critical point of higher order. It is straightforward to estimate for which modulation wave vector $q_{\mathrm{c}}$ this would happen (upon applying pressure for instance) if one assumes that $y=\mathrm{d} \alpha / \mathrm{d} T$ is constant. (This amounts to saying that $q^{2}$ varies linearly with temperature, which is not too far from reality at atmospheric pressure) one has the condition

$$
\frac{C \alpha_{\mathrm{c}}\left(T_{\mathrm{tr}}\right)}{\beta} y=1 / 2=-C y q_{\mathrm{c}}^{2}
$$

so that, using $S=4.2=\left(1+2 C y q\left(T_{\text {tr }}\right)\right)^{-1}$, we find

$$
q_{\mathrm{c}} \cong 0.16 \text {. }
$$

The experimental data available about thiourea under pressure $[2,6]$ indicates this would happen at about $2 \mathrm{kbar}$. The line of isolated critical points terminates at the point where it meets the line of tricritical points. Finally we note excellent agreement between theory and experiments around $T_{\lambda}(0)$. We find

$$
\begin{aligned}
\delta T(E) & =-\frac{25}{108}\left(T_{\mathrm{tr}}-T_{0}\right)\left(\frac{q\left(T_{\mathrm{tr}}\right)}{q\left(T_{\lambda}\right)}\right)^{8}\left(\frac{E}{E_{\mathrm{tr}}}\right)^{2} \\
& =-(1 \pm 0.2)\left(E / E_{\mathrm{tr}}\right)^{2} .
\end{aligned}
$$

It is interesting to compute at $E=0$ the temperature $T_{\mathrm{f}}$ of metastability of the ferroelectric phase and the temperature $T_{\mathrm{s}}$ of metastability of the modulated

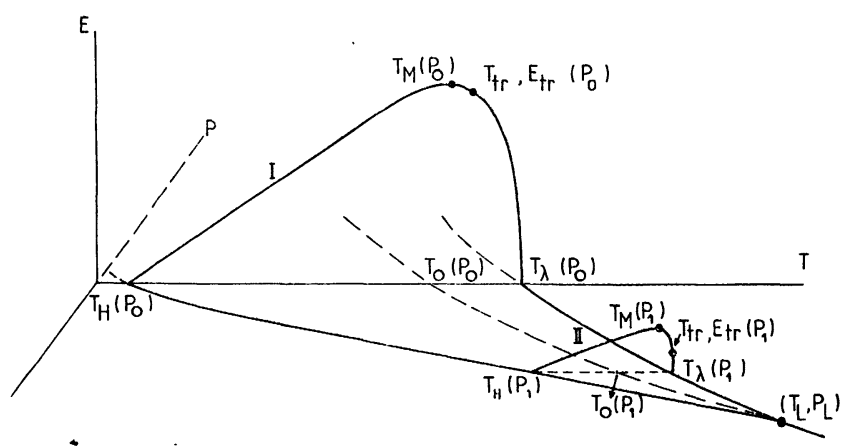

Fig. 1. - Curve $I$ is a schematic representation of the experimental results at atmospheric pressure. Curve II represents the phase diagram at a hypothetical negative pressure $P_{1}$ nearer to the Lifshitz point $\left(T_{\mathrm{L}}, P_{\mathrm{L}}\right)$. At $P_{1}$, the tricritical point is relatively further way from $T_{M}\left(P_{1}\right)$ (the isolated critical point) than at atmospheric pressure. The line of isolated critical points joins $T_{\mathrm{L}}\left(P_{\mathrm{L}}\right), T_{\mathrm{M}}\left(P_{1}\right)$, $T_{\mathrm{M}}\left(P_{0}\right)$. The line of tricritical points joins $T_{\mathrm{L}}\left(P_{2}\right), T_{\mathrm{tr}}\left(P_{1}\right), T_{\mathrm{tr}}\left(P_{0}\right)$. It separates the critical surface on the high temperature side from the surface of first-order transitions.

phase. According to the discussion in reference [4], we have

and

$$
\begin{aligned}
& T_{\mathrm{f}}=T_{0}(P)-0.25 C \frac{\alpha^{2}\left(T_{\mathrm{f}}\right)}{\beta} \\
& T_{\mathrm{s}}=T_{0}(P)-4.9 C \frac{\alpha^{2}\left(T_{\mathrm{s}}\right)}{\beta}
\end{aligned}
$$

a linear extrapolation for $\alpha$ from high temperatures yields $T_{\mathrm{f}} \cong 199 \mathrm{~K}$ and $T_{\mathrm{s}} \cong 184 \mathrm{~K}$. It is thus clear that the Landau theory described here accounts easily for the observed hysteresis effects of the order of $1 \sim 2 \mathrm{~K}$. We conclude that the Landau theory based on the functional described by equation (1) is a correct starting point for the physics of thiourea, which, in some sense appears, at atmospheric pressure, to be in the vicinity of a Lifshitz point, as shown in figure 1 . As evidenced by pressure measurements $[2,6]$, this Lifshitz point is a virtual one, at a negative pressure $P_{\mathrm{L}}=\sim-4.5 \mathrm{kbar}$ and $T_{\mathrm{L}} \simeq 290 \mathrm{~K}$.

The discussion of multiple Fourier components effects, the fine structure of the $q(T)$ curve, and in particular the pinning at $q=1 / 9$, and other pinnings under pressure $[2,6]$ should start from the recognition that the main temperature dependence of the modulation wave vector is imposed by the temperature dependence of the (negative) exchange stiffness constant $\alpha(T)$ in equation (1).

Acknowledgments. - We have benefited from the joint CNRS-CNPq scientific exchange program, which made our collaboration possible in Orsay and in Rio. One of us (P. L.) would like to thank Joana Siran for continuous interest and support while this work was completed, and the Physics Department in P.U.C. (Rio de Janeiro) for their friendship and hospitality. Illuminating discussions with $J$. Lajzerowicz are gratefully acknowledged. We thank J. P. Jamet for communicating his results and discussing them with us prior to publication. We have had pleasant and useful discussions with J. Ferré and A. H. Moudden. 


\section{References}

[1] JAMET, J. P., J. Physique Lett. 42 (1981) L-123.

[2] Denoyer, F. et al., J. Physique Lett., to be published.

[3] Moudden, A. H., Denoyer, F., Benort, J. P., Fitzgerald, W., Solid State Commun. 28 (1978) 575.

[4] Hornreich, R. M., Luban, M. and Strikman, S., Phys. Rev. Lett. 35 (1975) 1078.

Michelson, A., Phys. Rev. B 16 (1977) 577.
[5] Coutinho-Filho, M. D. and Marco de Moura, J. Mag. Mag. Mat. 15-18, I (1980) 433.

[6] Moudden, A. H., Thèse d'état, Orsay, unpublished (1980).

[7] Lederer, P., Mederros, J. T. N., Theodorou, G., Solid State Commun. 32 (1979) 1247.

[8] We thank J. Lajzerowicz for pointing this out to us. 